03

\title{
Математическая модель фракционирования малоконцентрированной дисперсной фазы суспензии в плоском вертикальном гидроклассификаторе
}

\author{
(C) A.В. Ряжских \\ Воронежский государственный технический университет, \\ 394026 Воронеж, Россия \\ e-mail: ryazhskihav@bk.ru
}

Поступило в Редакцию 9 января 2020 г.

В окончательной редакции 18 февраля 2020 г.

Принято к публикации 19 фревраля 2020 г.

\begin{abstract}
На основе гипотезы о непрерывном континууме и балансе потоков фаз предложена математическая модель переноса малоконцентрированной суспензии при отсутствии перемешивания в плоском вертикальном гравитационном классификаторе с ламинарным режимом течения несущей среды без ограничения на скорость седиментации частиц. Получены аналитические соотношения для расчета локальных счетных функций плотности распределения частиц по размерам. Вычислительный эксперимент подтвердил фракционирование монодисперсной суспензии и присутствие мелких частиц в „тяжелой“ фракции полидисперсных суспензий из-за малых значений скорости дисперсионной среды у „смоченных“ поверхностей гидроклассификатора. Результаты согласуются с известными экспериментальными данными и расчетами по классическим кинетическим моделям.
\end{abstract}

Ключевые слова: математическая модель, классификатор, суспензия, седиментация, дисперсная фаза, полидисперсность, функция распределения частиц по размерам.

DOI: $10.21883 / J T F .2020 .08 .49536 .3-20$

\section{Введение}

Сепарация фаз при течении гетерогенных сред в вертикальных аппаратах широко используется в химической и пищевой технологиях, в биохимической, нефтегазовой и металлургической промышленности, а также в экологии [1,2]. Ключевым параметром, влияющим на производительность оборудования, является скорость классификации одной фазы относительной другой [3], в результате чего происходит фракционирование частиц суспензии. В основе гравитационного фракционирования взвеси лежит процесс седиментации, основанный на действии силы тяжести и разнице плотностей фаз [4], который реализуется в различного типа гидроклассификаторах [5], причем эффективность их функционирования зависит от физико-химических характеристик суспензии, геометрических параметров оборудования и величин потоков фаз.

Моделирование явлений переноса в гидроклассификаторах обычно осуществляется в рамках двух подходов - эйлеро-эйлеровского и лагранжево-эйлеровского. Первый - наиболее часто применяемый - основан на гипотезе континуума, т.е. движение каждой фазы описывается уравнениями непрерывности и движения [6,7], причем идентификация межфазного взаимодействия остается нерешенной до конца проблемой. Другой заключается в использовании дискретной модели дисперсной фазы, которая отслеживает поведение каждой частицы, а гипотеза континуума формализует гидродинамику дисперсионной среды [8]. Использование такого подхода возможно в случае малых концентраций дисперсной фазы, так как увеличение числа частиц приводит к пропорциональному увеличению времени расчета. В последнее время стал также развиваться подход с применением стохастических методов [9].

Перечисленные стратегии требуют существенных усилий при проведении многовариантных вычислительных экспериментов, поэтому на проектных стадиях разработки гидроклассификаторного оборудования необходим альтернативный аналитический инструментарий оценок выбора диапазонов варьирования основных конструкционных и режимных характеристик, в основу которого должны быть положены модели, по крайне мере, в линейном приближении корректно описывающие физическую картину процессов в гидроклассификаторах. Наиболее часто используемая модель, удовлетворяющая таким требованиям, основана на кинетических балансовых соотношениях [10], однако ее затруднительно адаптировать на полидисперсный случай. Тем не менее дальнейшее ее развитие пошло по пути обобщения на случай счетного количества фракций с одновременным учетом их взаимодействия [11], но возможность определения локальной функции распределения частиц по размерам в сепарационных зонах гидроклассификаторов по-прежнему отсутствует. Решение такой задачи возможно в рамках гипотезы непрерывного континуума с использованием балансовых соотношений в дифференциальной форме и принципа суперпозиции концентрационных полей для малоконцентрированной дисперсной фазы [12]. 


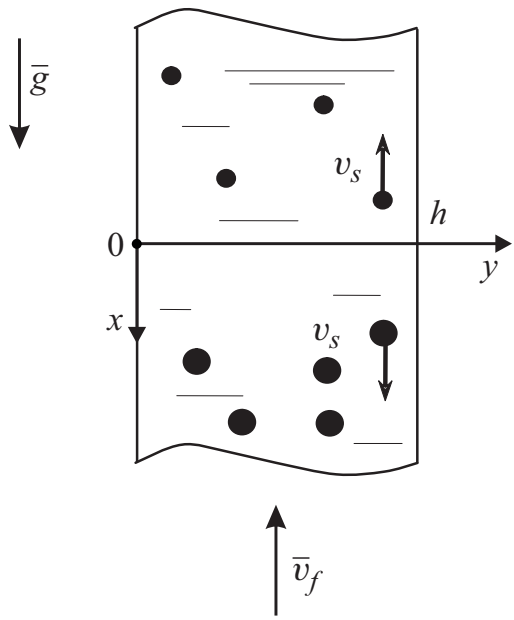

Рис. 1. Расчетная схема плоского вертикального гидроклассификатора.

\section{Постановка задачи}

В плоский вертикальный гидроклассификатор шириной $h[\mathrm{~m}]$ подается установившийся ламинарный поток ньютоновской несжимаемой среды с известной динамической вязкостью $\mu_{f}[\mathrm{~Pa} \cdot \mathrm{s}]$ и плотностью $\rho_{f}\left[\mathrm{~kg} / \mathrm{m}^{3}\right]$ со средней скоростью $\bar{v}_{f}[\mathrm{~m} / \mathrm{s}]$ в направлении, противоположном действию вектора силы тяжести $\bar{g}\left[\mathrm{~m} / \mathrm{s}^{2}\right]$ (рис. 1). В выбранной $2 D$-декартовой системе координат при $x=0$ диспергируется малоконцентрированная полидисперсная взвесь твердых частиц с текущим размером $l[\mathrm{~m}]$, плотностью $\rho_{s}\left[\mathrm{~kg} / \mathrm{m}^{3}\right]$, коэффициентом формы $k_{V}$ и с седиментационной скоростью $v_{s}[\mathrm{~m} / \mathrm{s}]$. Идеальные условия изотермического фракционирования дисперсной фазы таковы: осадок на стенках гидроклассификатора не образуется; перемешивание несущей среды, а также взаимодействие и кластеризация [13] частиц пренебрежимо малы, как и влияние начального гидродинамического участка; в связи с этим эффект Сегре-Зильберберга [14], а также силы Сафмана [15], Магнуса [16] и Боссе [17] не учитываются.

Для малоконцентрированной монодисперсной суспензии из рассмотрения баланса потоков фаз в характерном элементарном объеме и с учетом принятых допущений сформулирована начально-краевая задача для уравнения переноса

$$
\frac{\partial n(x, y, \tau)}{\partial \tau}=-u(y) \frac{\partial n(x, y, \tau)}{\partial x},
$$

с условиями отсутствия в начальный момент времени в вертикальном гидроклассификаторе частиц

$$
n(x, y, 0)=0 ;
$$

и постоянства их концентрации в месте диспергирования

$$
n(0, y, \tau)=n_{0}=\mathrm{const},
$$

где при $u(y)=v_{s}-v_{f}(y) \geq 0 \quad$ интегрирование системы (1)-(3) осуществляется в области
$\Omega^{+}(x, y)=\{0 \leq x<\infty, 0 \leq h \leq h\}$, а при $u(y)=v_{s}-$ $-v_{f}(y)<0-$ в области $\Omega^{-}(x, y)=\{-\infty<x \leq 0$, $0 \leq y \leq h\} ; \tau-$ текущее время [s]; $v_{f}(y)$ - локальная скорость несущей среды $[\mathrm{m} / \mathrm{s}] ; n(x, y, \tau)$ - счетная концентрация частиц $\left[\mathrm{m}^{-3}\right]$.

Суперпозиция концентрационных полей фракций, найденных из решения системы (1)-(3), позволяет определить локальные функции плотности распределения частиц по размерам.

\section{Анализ}

Вначале проанализирован случай монодисперсной суспензии. Скорость стабилизированного восходящего ламинарного течения в вертикальном плоском канале такова [18]:

$$
v_{f}(y)=\frac{h^{2}}{2 \mu_{f}}\left(-\frac{d p}{d x}+\rho_{f} g\right)\left[\left(\frac{y}{h}\right)^{2}-\frac{y}{h}\right],
$$

где $p-$ давление [Pa]. Связь седиментационного числа Рейнольдса $\mathrm{Re}_{s}=v_{s} l \rho_{f} / \mu_{f}$ с числом Архимеда $\mathrm{Ar}=l^{3} \rho_{f}\left(\rho_{s}-\rho_{f}\right) g / \mu_{f}^{2}$ для ламинарного $\left(10^{-4} \leq \operatorname{Re}_{s}<2\right)$, переходного $2 \leq \operatorname{Re}_{s} \leq 500$ и турбулентного $\left(\mathrm{Re}_{s}>500\right)$ режимов осаждения есть [19]

$$
\operatorname{Re}_{s}=\frac{\mathrm{Ar}}{18+0.61 \sqrt{\mathrm{Ar}}}
$$

откуда

$$
v_{s}=\frac{\mu_{f}}{l \rho_{f}} \cdot \frac{\mathrm{Ar}}{18+0.61 \sqrt{\mathrm{Ar}}} .
$$

Относительная скорость гидроклассификации, нормированная на среднюю скорость $\bar{u}=v_{s}-\bar{v}_{f}$, составляет

$$
U(Y)=\frac{u(y)}{\bar{u}}=[1-6 \mathrm{Rs} \cdot Y \cdot(1-Y)] /(1-\mathrm{Rs}),
$$

где $Y=y / h$, а безразмерный критерий

$$
\text { Rs }=-\frac{h^{2}}{12 \mu_{f}}\left(-\frac{d p}{d x}+\rho_{f} g\right) /\left(\frac{\mu_{f}}{l \rho_{f}} \cdot \frac{\mathrm{Ar}}{18+0.61 \sqrt{\mathrm{Ar}}}\right)
$$

характеризует величину отношения средней скорости восходящего потока несущей среды в вертикальном плоском гидроклассификаторе к скорости седиментации частиц размера $l$.

Безразмерное концентрационное поле, как результат интегрирования системы (1)-(3), имеет вид

$$
N(X, Y, \theta)= \begin{cases}1_{+}[\theta \cdot U(Y)-X], & X \geq 0 \text { при } U(Y) \geq 0 ; \\ 1_{+}[X-\theta \cdot U(Y)], & X<0 \text { при } U(Y)<0 ;\end{cases}
$$

где $\quad \theta=\bar{u} \tau / h ; \quad X=x / h ; \quad N(X, Y, \theta)=n(x, y, \theta) / n_{0}$; $1_{+}[\ldots]-$ ассиметричная единичная функция Хэвисайда [20]. Упрощение выражения (8) с использованием модельных представлений о движении 


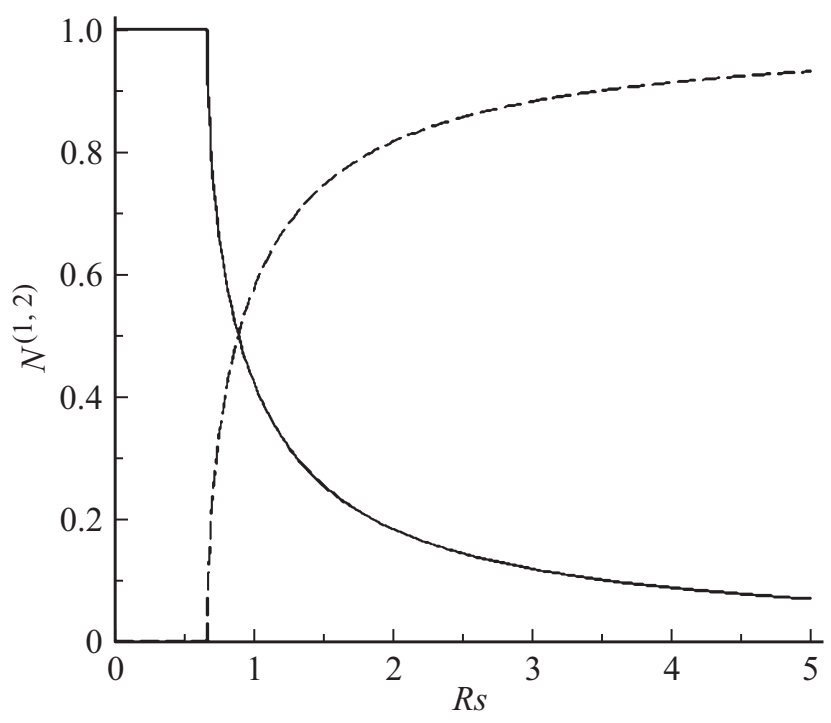

Pис. 2. Фракционирование монодисперсной суспензии в вертикальном гидроклассификаторе: - „нижний“ отбор частиц; - — „верхний“ отбор частиц.

несущей среды в режиме идеального вытеснения приводит к существенной неточности, особенно в случае геометрически компактных гидроклассификаторов. Из рассмотрения режима стационарного функционирования гидроклассификатора, т.е. $X / \theta \ll 1$, и проведения процедуры осреднения по поперечной координате $Y$, из (8) следуют выражения для концентраций частиц в „нижней“ и „верхней“ зонах отбора соответственно

$$
\begin{gathered}
N^{(1)}= \begin{cases}1-\sqrt{1}-2 /(3 \cdot \mathrm{Rs}), & \text { при Rs } \geq 2 / 3 ; \\
1, & \text { при } 0 \leq \mathrm{Rs}<2 / 3,\end{cases} \\
N^{(2)}= \begin{cases}\sqrt{1}-2 /(3 \cdot \mathrm{Rs}), & \text { при Rs } \geq 2 / 3 ; \\
1, & \text { при } 0 \leq \mathrm{Rs}<2 / 3,\end{cases}
\end{gathered}
$$

Анализ показывает (рис. 2), что при учете реального профиля несущей среды наблюдается процесс псевдофракционирования монодисперсной взвеси из-за неравномерности профиля скорости. Если определить коэффициент сепарации, как $\xi=N^{(1)} / N^{(2)}$, и сравнить его значения с результатами пилотного эксперимента [21] по осаждению монодисперсных частиц карбоната кальция размеров $10,25,50,100$ и $250 \mu \mathrm{m}$ в восходящем водном потоке в вертикальном гидроклассификаторе со скоростью $\bar{v}_{f}=7.5 \cdot 10^{-3} \mathrm{~m} / \mathrm{s}$ (рис. 3 ), то некоторое отличие для фракции мелких частиц может быть объяснено иным условием входа несущей среды (с разворотом потока), вызывающим отклонение от ламинарного течения дисперсионной фазы [22].

Пусть при $X=0$ в гидроклассификатор диспергируется полидисперсная взвесь с функцией распределения $f_{0}(l)\left[\mathrm{m}^{-4}\right]$ и со среднечисленным размером

$$
\bar{l}_{N}=\int_{0}^{\infty} l f_{0}(l) d l / \int_{0}^{\infty} f_{0}(l) d l
$$

Введение безразмерных параметров:

$$
\bar{\theta}=\bar{u}\left(\bar{l}_{N}\right) \tau / h ; \quad L=l / \bar{l}_{N} ; \quad \overline{\mathrm{Ar}}=\bar{l}_{N}^{3} \rho_{f}\left(\rho_{s}-\rho_{f}\right) g / \mu_{f}^{2}
$$

и применение принципа суперпозиции концентрационных полей к (8) дает выражение для локальной безразмерной функции распределения частиц по относительным размерам

$$
\begin{aligned}
& F_{N}(X, Y, L, \bar{\theta})=F_{N_{0}}(L) \\
& \times \begin{cases}1_{+}[\bar{\theta} \cdot U(Y)-X], & X \geq 0 \text { при } U(Y) \geq 0 ; \\
1_{+}[X-\bar{\theta} \cdot U(Y)], & X<0 \text { при } U(Y)<0 ;\end{cases}
\end{aligned}
$$

где $\quad F_{N}(X, Y, L, \bar{\theta})=\bar{l}_{N} f(x, y, l, \tau) / \int_{0}^{\infty} f(x, y, k, \tau) d l$; $F_{N_{0}(L)}=\bar{l}_{N} f_{0}(l) / \int_{0}^{\infty} f_{0}(l) d l$

$$
\begin{aligned}
& U(Y, L)=\left(L^{2} \frac{18+0.61 \sqrt{\overline{\mathrm{Ar}}}}{18+0.61 L \sqrt{L \cdot \overline{\mathrm{Ar}}}}\right)^{2} \\
& \times\left[1-6 \overline{\mathrm{Rs}} Y(1-Y) /\left(L^{2} \frac{18+0.61 \sqrt{\overline{\mathrm{Ar}}}}{18+0.61 L \sqrt{L \cdot \overline{\mathrm{Ar}}}}\right)\right]
\end{aligned}
$$

$\overline{\mathrm{Rs}}=-\frac{h^{2}}{12 \mu_{f}}\left(-\frac{d p}{d x}+\rho_{f} g\right) /\left(\frac{\mu_{f}}{\bar{l}_{N} \rho_{f}} \cdot \frac{\overline{\mathrm{Ar}}}{19+0.61 \sqrt{\overline{\mathrm{Ar}}}}\right)$,

$f(x, y, l, \tau), f_{0}(l)$ - локальная и диспергируемая счетные функции распределения частиц по размерам $\left[\mathrm{m}^{-4}\right]$.

Для оценки фракционного состава суспензии по координате $X$ необходима осредненная по поперечному сечению классификатора безразмерная нормированная

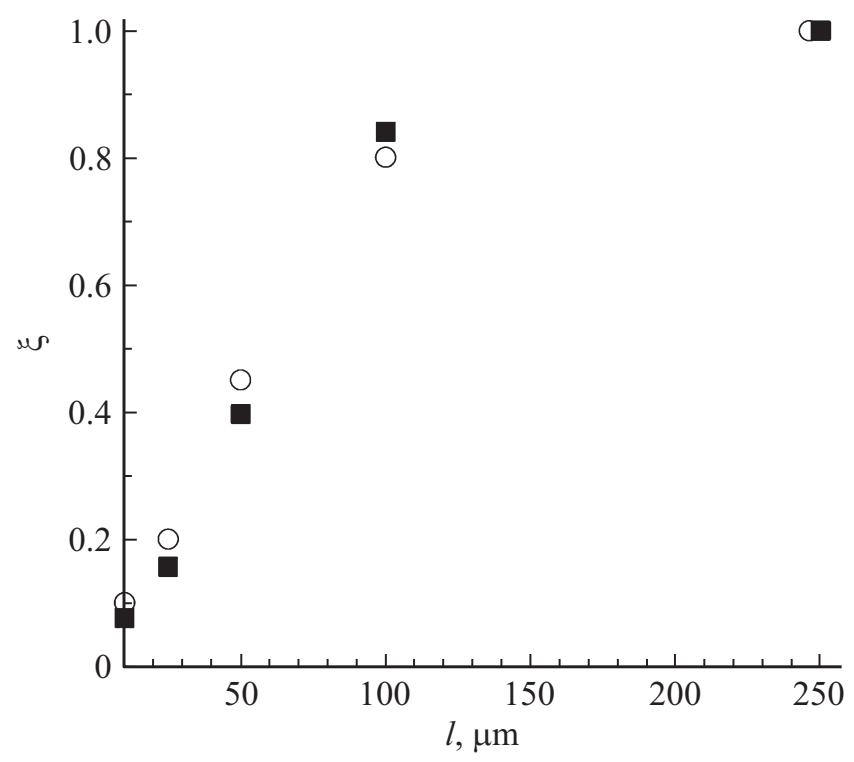

Рис. 3. Коэффициент сепарации монодисперсной взвеси: оэксперимент [21]; $\mathbf{\square}$ - расчет по модели. 
функция плотности распределения частиц по относительным размерам

$$
\bar{F}_{N}(X, L, \bar{\theta})=\int_{0}^{1} F_{N}(X, Y, L, \bar{\theta}) d Y .
$$

Если в (9) ввести систему обозначений

$$
\begin{aligned}
& C=B / A ; \quad A=a b ; \quad B=a-X, \\
& a=\bar{\theta}\left(L^{2} \frac{18+0.61 \sqrt{\overline{\mathrm{Ar}}}}{18+0.61 L \sqrt{L \cdot \overline{\mathrm{Ar}}}}\right)^{2}, \\
& b=6 \overline{\mathrm{Rs}}\left(L^{2} \frac{18+0.61 \sqrt{\overline{\mathrm{Ar}}}}{18+0.61 L \sqrt{L \cdot \overline{\mathrm{Ar}}}}\right),
\end{aligned}
$$

то результат интегрирования (10) будет

для $X \geq 0$ и $U(Y, L) \geq 0$

$$
\bar{F}_{N}^{(1)}(X, L, \bar{\theta})=\bar{F}_{N_{0}}(L) \begin{cases}1-Y_{1}, & \text { при } C<0 ; \\ 1-Y_{1}+Y_{2}, & \text { при } 0 \leq C \leq 1 / 4 ; \\ 0, & \text { при } C>1 / 4,\end{cases}
$$

$$
\text { для } X<0 \text { и } U(Y, L)<0
$$$$
\bar{F}_{N}^{(2)}(X, L, \bar{\theta})=\bar{F}_{N_{0}}(L) \begin{cases}Y_{1}, & \text { при } C<0 ; \\ Y_{1}-Y_{2}, & \text { при } 0 \leq C \leq 1 / 4 ; \\ 0, & \text { при } C>1 / 4,\end{cases}
$$

где $Y_{1,2}=1 / 2 \pm \sqrt{1 / 4-C}$.

Пусть $H_{1}=h_{1} / h$ и $H_{2}=h_{2} / h$ - относительные аксиальные размеры зон классификации для $X \geq 0$ и $X<0$ соответственно, тогда оценка времени наступления стационарного процесса фракционирования может быть найдена из условия, например, $1 \%$-го отклонения от нестационарного, т.е.

$$
\begin{gathered}
\bar{\theta}=100 \max \left(H_{1}, H_{2}\right) \\
\text { или } \tau=100(18+0.61 \sqrt{\overline{\mathrm{Ar}}}) \cdot \frac{\bar{l}_{N} \rho_{f}}{\mu_{f} \overline{\mathrm{Ar}}} \max \left(h_{1}, h_{2}\right) .
\end{gathered}
$$

Практический интерес представляет случай, когда $H_{1,2} \ll \bar{\theta}$, поэтому из (11) и (12) следуют соотношения: для $X \geq 0$

$$
\begin{aligned}
& \bar{F}_{N}^{(1)}(L)=F_{N_{0}}(L) \\
& \times\left\{\begin{array}{lr}
1-\sqrt{1-\frac{2 L^{2}}{2 \overline{\mathrm{Rs}}}} \frac{18+0.61 \sqrt{\overline{\mathrm{Ar}}}}{18+0.61 L \sqrt{L \cdot \overline{\mathrm{Ar}}}}, & \text { или } 0 \leq L \leq L^{*} ; \\
1, & \text { или } L>L^{*} ;
\end{array}\right.
\end{aligned}
$$

$$
\begin{aligned}
& \text { для } X<0 \\
& \bar{F}_{N}^{(2)}(L)=F_{N_{0}}(L) \\
& \times\left\{\begin{array}{lc}
\sqrt{1-\frac{2 L^{2}}{2 \overline{\mathrm{Rs}}}} \frac{18+0.61 \sqrt{\overline{\mathrm{Ar}}}}{18+0.61 L \sqrt{L \cdot \overline{\mathrm{Ar}}}}, & \text { или } 0 \leq L \leq L^{*} ; \\
0, & \text { или } L>L^{*} ;
\end{array}\right.
\end{aligned}
$$

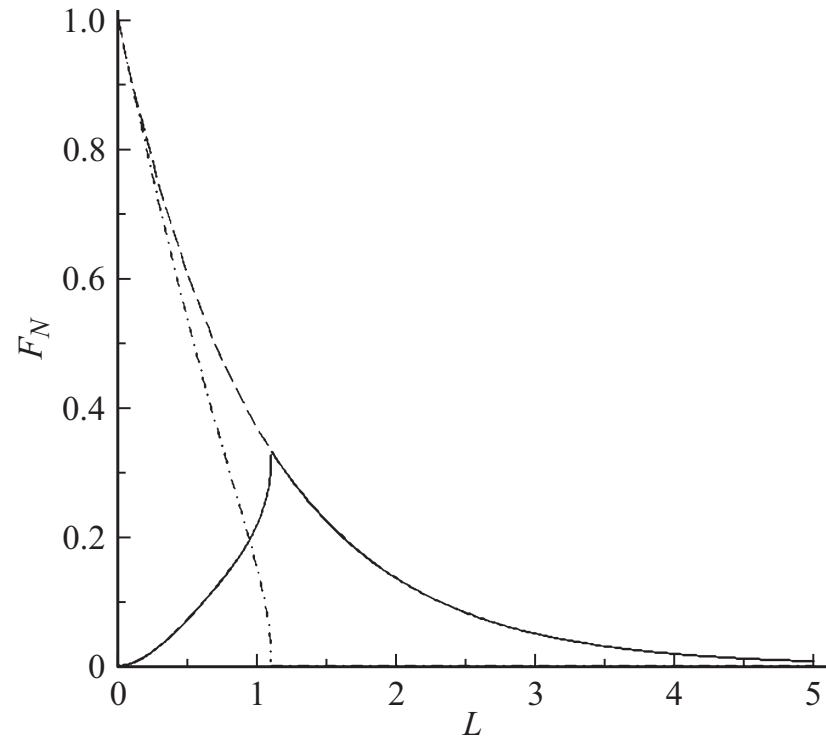

Рис. 4. Функции распределения частиц по размерам в классификаторе при $\overline{\mathrm{Ar}}=10$ и $\overline{\mathrm{Rs}}=0.8:-$ исходная; - „тяжелых“ фракций; - „легких“ фракций.

где $L^{*}-$ корень уравнения

$$
\frac{2 L^{2}}{3 \mathrm{Rs}} \frac{18+0.61 \sqrt{\cdot \overline{\mathrm{Ar}}}}{18+0.61 L \sqrt{L \cdot \overline{\mathrm{Ar}}}}=1 .
$$

Для исходной экспоненциальной функции распределения $\left(F_{N_{0}}=\exp (-L), L \in[0.10]\right)$, которой наиболее часто подчиняются природные дисперсные материалы [23], вид функций распределения „тяжелых“ (14) и „легких“ (15) фракций на выходе из классификатора (рис. 4) подтверждает качественную адекватность предложенной модели процессу фракционирования. Детальный количественный анализ результирующих функций проведен с помощью их числовых характеристик с предварительным переформатированием (14) и (15) к нормированному виду

$$
\bar{F}_{N_{n}}^{(1,2)}(L)=\bar{F}_{N_{n}}^{(1,2)}(L) / \int_{0}^{\infty} \bar{F}_{N}^{(1,2)}(L) d L,
$$

для которых определены относительные среднечисленные размеры частиц

$$
\bar{L}^{(1,2)}=\int_{0}^{\infty} L \bar{F}_{N_{n}}^{(1,2)}(L) d L
$$

и среднеквадратические отклонения от них

$$
\sigma^{(1,2)}=\left[\int_{0}^{\infty}\left(L-\bar{L}^{(1,2)}\right)^{2} \bar{F}_{N_{n}}^{(1,2)}(L) d L\right]^{1 / 2} .
$$

На всех гидродинамических режимах седиментации (рис. 5) для „тяжелых“ фракций наблюдается максимум относительно среднего размера частиц, когда 

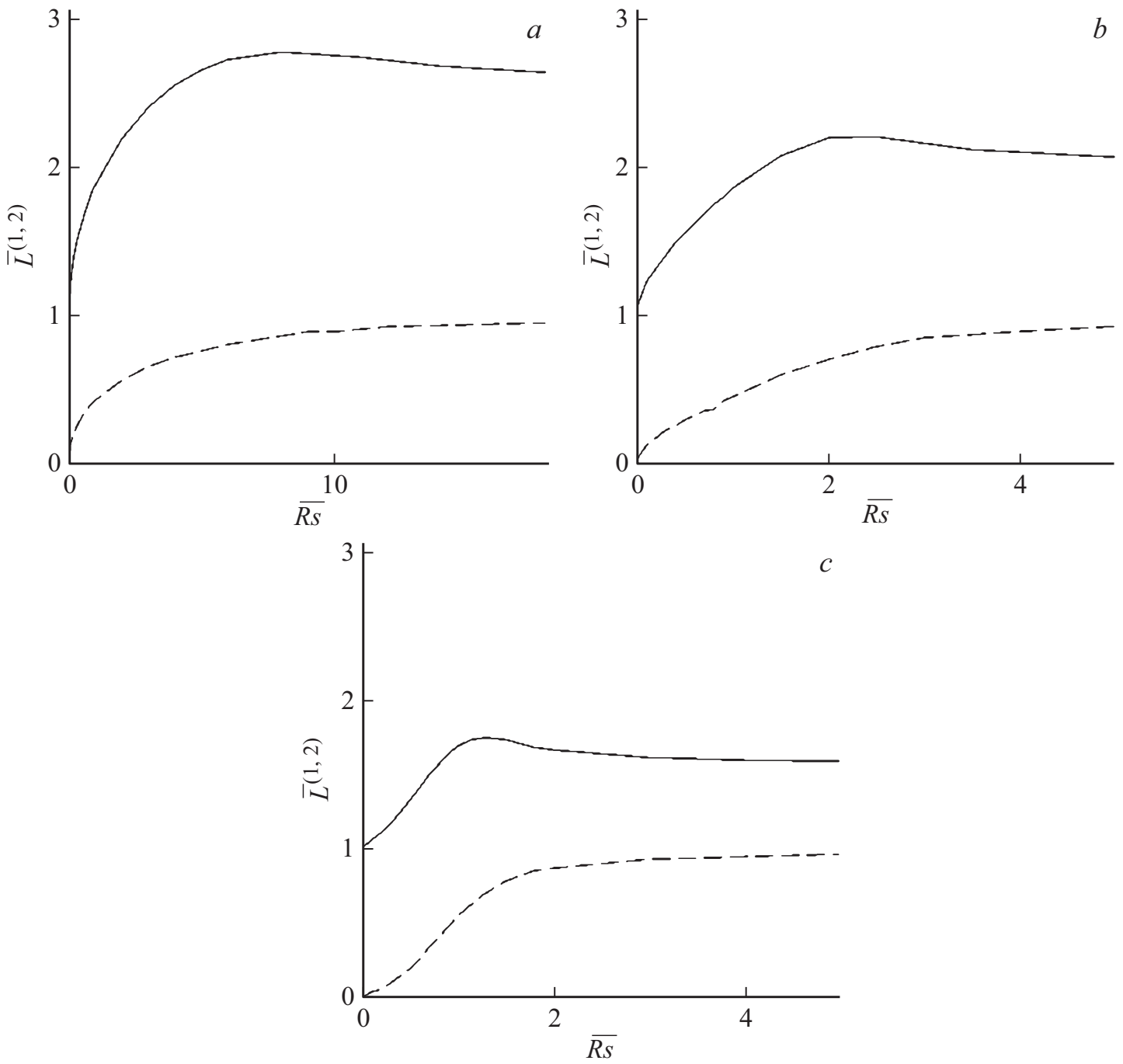

Рис. 5. Изменение относительных среднечисленных размеров частиц „тяжелой“ и „легкой“ фракций в зависимости от отношения средней скорости несущей среды и скорости седиментации частиц среднечисленного размера исходной функции распределения, диспергируемой в классификатор, для различных режимов седиментации: $a-$ ламинарный $(\overline{\mathrm{Ar}}=10) ; b-$ переходный $\left(\overline{\mathrm{Ar}}=10^{3}\right)$; $c$ - турбулентный $\left(\overline{\mathrm{Ar}}=10^{6}\right) ;-\bar{L}^{(1)} ;--\bar{L}^{(2)}$.

Таблица 1. Среднеквадратическое отклонение от среднечисленных относительных раз меров частиц „тяжелой“ и ,легкой“ фракций для различных режимов седиментации

\begin{tabular}{l|l|l|l|l|l|l}
\hline Режимы & \multicolumn{2}{|c|}{ Ламинарный } & \multicolumn{2}{|c|}{ Переходный } & \multicolumn{2}{|c}{ Турбулентный } \\
\hline$\overline{\mathrm{Ar}}$ & \multicolumn{2}{|c|}{10} & \multicolumn{2}{|c|}{$10^{3}$} & \multicolumn{2}{|c}{$10^{6}$} \\
\hline$\overline{\mathrm{Rs}}$ & $\sigma^{(1)}$ & $\sigma^{(2)}$ & $\sigma^{(1)}$ & $\sigma^{(2)}$ & $\sigma^{(1)}$ & $\sigma^{(2)}$ \\
\hline 0.2 & 1 & 0.14 & 1.00 & 0.11 & 0.99 & 0.04 \\
0.6 & 1.02 & 0.24 & 1.02 & 0.23 & 1.03 & 0.22 \\
1 & 1.05 & 0.3 & 1.07 & 0.34 & 1.18 & 0.46 \\
3 & 1.2 & 0.52 & 1.39 & 0.81 & 1.24 & 0.94 \\
5 & 1.36 & 0.66 & 1.31 & 0.92 & 1.21 & 0.97
\end{tabular}

средняя скорость несущей среды превосходит скорость седиментации среднечисленного размера частиц исход- ной функции распределения, причем с увеличением $\overline{\mathrm{Ar}}$ величина $\overline{\mathrm{Rs}}$, соответствующая этому максимуму, уменьшается. Для „легких“ фракций такого эффекта не обнаруживается. Это может быть объяснено тем, что в пристеночной области скорость течения несущей среды мала и поэтому в $\bar{F}_{N_{n}}^{(1)}$ присутствуют все фракции твердой фазы, а для „легкой“ фракции увеличение $\bar{L}^{(2)}$ с ростом $\overline{\mathrm{Rs}}$ является монотонным. Отметим, что для обеих фракций при $\overline{\mathrm{Rs}} \gg 1$ наблюдается асимптотика, и при одних и тех же $\overline{\mathrm{Rs}}$ величины $\bar{L}^{(1,2)}$ уменьшаются при переходе от ламинарного режима седиментации через переходный режим и к турбулентному. Как и следовало ожидать, особенности изменения среднеквадратического отклонения аналогичны изменениям среднечисленных относительных размеров частиц „тяжелых“ и „легких“ фракций (табл. 1). 


\section{Пример}

Воспользуемся результатами оценки разделительной способности вертикальных гравитационных гидроклассификаторов [24] в рамках кинетических балансовых соотношений с использованием дискретной функции распределения частиц по размерам (табл. 2) при $\rho_{s}=2900 \mathrm{~kg} / \mathrm{m}^{3} ; \rho_{f}=1100 \mathrm{~kg} / \mathrm{m}^{3} ; \mu_{f}=1 \cdot 10^{-3} \mathrm{~Pa} \cdot \mathrm{s}$; $\bar{v}_{f}=6 \cdot 10^{-4} \mathrm{~m} / \mathrm{s}$. По исходной функции распределения, учитывая тот факт, что

$$
f_{V}(L)=f_{M}(L)=d M(l) /(M d l)
$$

где $d M(l), M-$ массы фракции частиц размера от $l$ до $l+d l$ и всех частиц в диспергируемой суспензии, вычис-

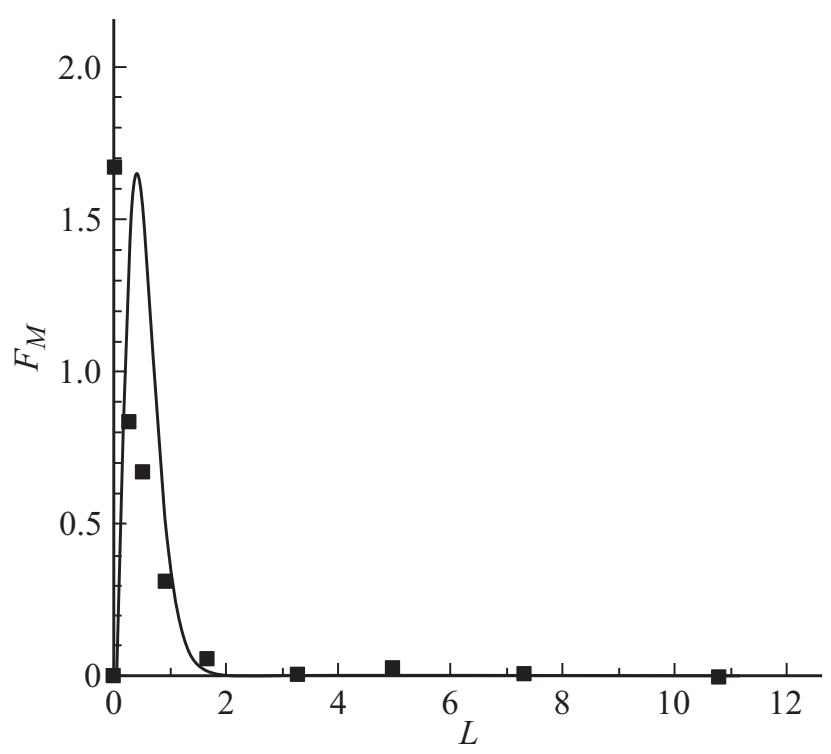

Рис. 6. К аппроксимации функции распределения: исходная; - аппроксимация.

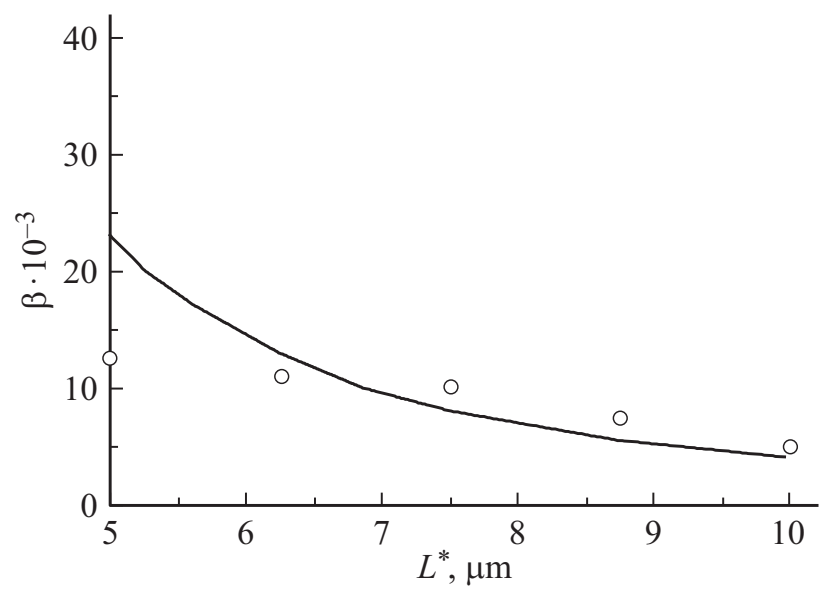

Рис. 7. Объемная доля „легкой“ фракции: о — данные из [24]; - расчет.
Таблица 2. Функция распределения частиц по размерам

\begin{tabular}{c|c}
\hline$\Delta l, \mu \mathrm{m}$ & $f_{V}=\frac{\Delta V}{V \Delta l}, \mathrm{~m}^{-1}$ \\
\hline $0-5$ & 0.030000 \\
$5-10$ & 0.030000 \\
$10-20$ & 0.015000 \\
$20-40$ & 0.012000 \\
$40-63$ & 0.005600 \\
$63-125$ & 0.001100 \\
$125-240$ & 0.000240 \\
$240-315$ & 0.000470 \\
$315-500$ & 0.000200 \\
$500-700$ & 0.000015
\end{tabular}

лен среднемассовый размер полидисперсных частиц

$$
\bar{l}_{M}=f_{0}^{\infty} l f_{M}(L) d l \approx 55.89 \mu \mathrm{m} .
$$

Безразмерная нормированная массовая фракция плотности распределения частиц по размерам (рис. 6) аппроксимирована соотношением

$$
F_{M}(L)=420 L^{3} \exp (-7 L),
$$

что позволило, используя связь

$$
F_{N_{n}}(L)=F_{M}(L) /\left\{L^{3}\left[\int_{0}^{-\infty} L^{-2} F_{M}(L) d L\right]^{2}\right\},
$$

найти $F_{N_{n}}(L)=\exp (-L)$, где $\bar{L}_{N}=8 \mu \mathrm{m}$. Результаты расчета объемной доли ,легкой“ фракции (рис. 7)

$$
\beta=\int_{0}^{L^{*}} \bar{F}_{N_{n}}^{(2)}(L) d L\left[\int_{0}^{L^{*}} L \bar{F}_{N_{n}}^{(2)}(L) d L\right]^{3}
$$

подтвердили корректность принятых допущений и адекватность модели.

\section{Заключение}

Предложенная математическая модель фракционирования малоконцентрированной взвеси в плоском гравитационном вертикальном гидроклассификаторе во всех режимах седиментации, учитывающая неравномерность профиля скорости несущей среды при ламинарном течении, позволяет объяснить наличие мелких частиц в „тяжелых“ фракциях и псевдоклассификацию монодисперсной взвеси, а также прогнозировать дисперсный состав в любой локализации рабочего объема классификатора и выбрать при его проектировании геометрические характеристики в зависимости от расходных и физикохимических параметров суспензии.

\section{Конфликт интересов}

Автор заявляет, что у него нет конфликта интересов. 


\section{Список литературы}

[1] Affa A., Razzak S.A., Nigam K.D., Zhu J.-X. // Ind. Eng. Chem. Res. 2009. Vol. 49. N 9. P. 7876-7882.

[2] Polchar R.R., Shilapurain V. // Particuology. 2017. Vol. 31. P. 59-68.

[3] Барский М.Д., Ревнивцев В.И., Соколкин Ю.В. Гравитационная классификация зернистых материалов. М.: Недра, 1974. 232 c.

[4] Goula A.M., Kostoglu M., Karapatsios T.D., Zouboulis A.L. // Chem. Eng. J. 2007. Vol. 140. P. 110-121.

[5] Farrow J.B., Fawell P.D., Johnston R.R.M., Nguyen T.B., Rudman M., Simic K., Swift J.D. // Chem. Eng. J. 2000. Vol. 180. P. 149-155.

[6] Cheng Y., Zhu J.-X. // Canad. Chem. Eng. 2005. Vol. 83. N 2. P. $177-185$.

[7] Чиркун Д.И., Саевич Н.П., Левданский А.Э., Ярмольник С.В. // Труды БГТУ. 2017. Сер. 2. № 2. С. 190-194.

[8] Di Renzo A., Cello F.D., Maio F.P. // Chem. Eng. Sci. 2011. Vol. 66. N 13. P. 2945-2958.

[9] Yongli M., Mingyan L., Yuan Z. // Chem. Eng. Sci. 2011. Vol. 66. N 13. P. 2945-2958.

[10] Nasr-El-Din H., Masliyah J.H., Naudakumar K., Law H.-S. // Chem. Eng. Sci. 1988. Vol. 43. N 13. P. 3225-3234.

[11] Berman Y., Tamir A. // Chem. Eng. Sci. 2003. Vol. 58. P. 2089-2102.

[12] Островский Г.М. Прикладная механика неоднородных сред. СПб.: Наука, 2000. 359 с.

[13] Лугуманов Т.T., Кулешов В.С. // Тр. Ин-та механики им. Р.Р. Мавлютова УНЦ РАН. 2014. Вып. 10. С. 66-72.

[14] Serge G., Silberberg A. // Nature. 1961. Vol. 189. P. 209-210.

[15] Saffman P.G. // J. Fluid Mech. 1965. Vol. 22. P. 385-400.

[16] Яценко В.П. // Физика аэродисперсных систем. 2002. Т. 39. C. 240-248.

[17] Губайдуллин Д.А., Осипов П.П. // Актуальные проблемы механики сплошной среды. К 20-летию ИММ КазНЦ РАН. Казань: Фолиант, 2011. Т. 1. С. 82-97.

[18] Берд Р., Стьюарт В., Лайтфут Е. Явления переноса. М.: Химия, 1974. $688 \mathrm{c}$.

[19] Дытнерский Ю.И. Процессы и аппараты химической технологии. Ч. 1. М.: Химия, 2002. 400 с.

[20] Корн Г.А., Корн Т.М. Справочник по математике для научных работников и инженеров. М.: Наука, 1974. 832 с.

[21] Luna F.D.T., Silva A.G., Fukumasu N.K., Bazan O., Gouveia J.H.A., Moraes Jr.D., Yanagihara J.I., Vianna Jr. A.S. // Chem. Eng. J. 2019. Vol. 362. P. 712-720.

[22] Доманский И.В., Давыдов И.В., Боровинский В.П. // Цветные металлы. 2000. № 1. С. 25-27.

[23] Гаель А.Г., Смирнова Л.Ф. Пески и песчаные почвы. М.: ГEOC, 1999. $252 \mathrm{c}$.

[24] Новый справочник химика и технолога. Процессы и аппараты химической технологий. Ч.ІІ. СПб.: НПО Профессионал, 2006. 916 с. 